\title{
The structure of fractional spaces generated by a two-dimensional elliptic differential operator and its applications
}

\author{
Allaberen Ashyralyev ${ }^{1,2}$, Sema Akturk' and Yasar Sozen ${ }^{1,3^{*}}$
}

\section{"Correspondence:}

ysozen@fatih.edu.tr;

ysozen@hacettepe.edu.tr

'Department of Mathematics, Fatih

University, 34500 Buyukcekmece,

Istanbul, Turkey

${ }^{3}$ Present address: Hacettepe Universitesi, Fen Fakultesi,

Matematik Bolumu, 06800 Beytepe, Ankara, Turkey

Full list of author information is

available at the end of the article

\begin{abstract}
We consider the two-dimensional differential operator

$\operatorname{Au}\left(x_{1}, x_{2}\right)=-a_{11}\left(x_{1}, x_{2}\right) u_{x_{1} x_{1}}\left(x_{1}, x_{2}\right)-a_{22}\left(x_{1}, x_{2}\right) u_{x_{2} x_{2}}\left(x_{1}, x_{2}\right)+\sigma u\left(x_{1}, x_{2}\right)$ defined on

functions on the half-plane $\Omega=\mathbb{R}^{+} \times \mathbb{R}$ with the boundary conditions $u\left(0, x_{2}\right)=0$,

$x_{2} \in \mathbb{R}$, where $a_{i i}\left(x_{1}, x_{2}\right), i=1,2$, are continuously differentiable and satisfy the uniform

ellipticity condition $a_{11}^{2}\left(x_{1}, x_{2}\right)+a_{22}^{2}\left(x_{1}, x_{2}\right) \geq \delta>0, \sigma>0$. The structure of the

fractional spaces $E_{\alpha}\left(A, C^{\beta}(\Omega)\right)$ generated by the operator $A$ is investigated. The

positivity of $A$ in Hölder spaces is established. In applications, theorems on

well-posedness in a Hölder space of elliptic problems are obtained.
\end{abstract}

MSC: 35J25; 47E05; 34B27

Keywords: positive operator; fractional spaces; Green's function; Hölder spaces

\section{Introduction}

It is well known that (see, for example, [1-3] and the references therein) various classical and non-classical boundary value problems for partial differential equations can be considered as an abstract boundary value problem for an ordinary differential equation in a Banach space with a densely defined unbounded operator. The importance of the positivity property of the differential operators in a Banach space in the study of various properties for partial differential equations is well known (see, for example, [4-7] and the references therein). Several authors have investigated the positivity of a wider class of differential and difference operators in Banach spaces (see [8-18] and the references therein).

Let us give the definition of positive operators and introduce the fractional spaces and preliminary facts that will be needed in the sequel.

The operator $A$ is said to be positive in $E$ if its spectrum $\sigma(A)$ lies inside of the sector $S$ of the angle $\phi, 0<2 \phi<2 \pi$, symmetric with respect to the real axis, and the following estimate (see, for example, $[6,19]$ )

$$
\left\|(A-\lambda)^{-1}\right\|_{E \rightarrow E} \leq \frac{M(\phi)}{1+|\lambda|}
$$

holds on the edges $S_{1}(\phi)=\left\{\rho e^{i \phi}: 0 \leq \rho<\infty\right\}, S_{2}(\phi)=\left\{\rho e^{-i \phi}: 0 \leq \rho<\infty\right\}$ of $S$, and outside of the sector $S$. The infimum of all such angles $\phi$ is called the spectral angle of the positive operator $A$ and is denoted by $\phi(A, E)$. We say that $A$ is a strongly positive operator in $E$ if $\phi(A, E)<\pi / 2$.

O2014 Ashyralyev et al.; licensee Springer. This is an Open Access article distributed under the terms of the Creative Commons Attribution License (http://creativecommons.org/licenses/by/2.0), which permits unrestricted use, distribution, and reproduction in any medium, provided the original work is properly cited. 
Throughout the article, $M$ indicates positive constants which may differ from time to time, and we are not interested to precise. If the constant depends only on $\alpha, \beta, \ldots$, then we will write $M(\alpha, \beta, \ldots)$.

With the help of the positive operator $A$, we introduce the fractional space $E_{\beta}=E_{\beta}(E, A)$ $(0<\beta<1)$, consisting of all elements $v \in E$ for which the norm

$$
\|v\|_{E_{\beta}}=\sup _{\lambda>0} \lambda^{\beta}\left\|A(\lambda+A)^{-1} v\right\|_{E}+\|v\|_{E}
$$

is finite.

Theorem 1 [20] Let $\frac{1}{p}+\frac{1}{q}=1, p>1$, and let $f, g: \mathbb{R}^{+} \rightarrow \mathbb{R}$ be any two nonnegative integrable functions such that $0<\int_{0}^{\infty} f^{p}(x) d x<\infty$ and $0<\int_{0}^{\infty} g^{q}(y) d y<\infty$. Then the following Hilbert's inequality holds:

$$
\int_{0}^{\infty} \int_{0}^{\infty} \frac{f(x) g(y)}{x+y} d x d y<\pi \csc \left(\frac{\pi}{p}\right)\left(\int_{0}^{\infty} f^{p}(x) d x\right)^{\frac{1}{p}}\left(\int_{0}^{\infty} g^{q}(y) d y\right)^{\frac{1}{q}}
$$

Danelich in [12] considered the positivity of a difference analog $A_{h}^{x}$ of the $2 m$ th-order multi-dimensional elliptic operator $A^{x}$ with dependent coefficients on semi-spaces $\mathbb{R}^{+} \times$ $\mathbb{R}^{n-1}$.

The structure of fractional spaces generated by positive multi-dimensional differential and difference operators on the space $\mathbb{R}^{n}$ in Banach spaces has been well investigated (see [21-23] and the references therein).

In papers [19, 24-27] the structure of fractional spaces generated by positive one-dimensional differential and difference operators in Banach spaces was studied. Note that the structure of fractional spaces generated by positive multi-dimensional differential and difference operators with local and nonlocal conditions on $\Omega \subset \mathbb{R}^{n}$ in Banach spaces $C(\Omega)$ has not been well studied.

In the present paper, we study the structure of fractional spaces generated by the twodimensional differential operator

$$
A u\left(x_{1}, x_{2}\right)=-a_{11}\left(x_{1}, x_{2}\right) u_{x_{1} x_{1}}\left(x_{1}, x_{2}\right)-a_{22}\left(x_{1}, x_{2}\right) u_{x_{2} x_{2}}\left(x_{1}, x_{2}\right)+\sigma u\left(x_{1}, x_{2}\right),
$$

defined over the region $\mathbb{R}_{+}^{2}=\mathbb{R}^{+} \times \mathbb{R}$ with the boundary condition $u\left(0, x_{2}\right)=0, x_{2} \in \mathbb{R}$. Here, the coefficients $a_{i i}\left(x_{1}, x_{2}\right), i=1,2$, are continuously differentiable and satisfy the uniform ellipticity

$$
a_{11}^{2}\left(x_{1}, x_{2}\right)+a_{22}^{2}\left(x_{1}, x_{2}\right) \geq \delta>0
$$

and $\sigma>0$.

Following the paper [12], passing limit when $h \rightarrow 0$ in the special case $m=1$ and $n=2$, we get that there exists the inverse operator $(A+\lambda)^{-1}$ for all $\lambda \geq 0$ and the following formula

$$
(A+\lambda)^{-1} f\left(x_{1}, x_{2}\right)=\int_{0}^{\infty} \int_{-\infty}^{\infty} G\left(x_{1}, x_{2}, p, s, \lambda\right) f(p, s) d s d p
$$


holds, where $G\left(x_{1}, x_{2}, p, s, \lambda\right)$ is the Green function of differential operator (1). Moreover, the following estimates

$$
\begin{aligned}
& \left|G\left(x_{1}, x_{2}, p, s, \lambda\right)\right| \\
& \quad \leq C \exp \left\{-a(\lambda+1)^{1 / 2}\left(\left|x_{1}-p\right|+\left|x_{2}-s\right|\right)\right\} \\
& \quad \times\left(1+\ln \left\{1+\left((\lambda+1)^{1 / 2}\left(\left|x_{1}-p\right|+\left|x_{2}-s\right|\right)\right)^{-1}\right\}\right),
\end{aligned}
$$

and

$$
\begin{aligned}
& \left|G_{x_{1}}\left(x_{1}, x_{2}, p, s, \lambda\right)\right|,\left|G_{x_{2}}\left(x_{1}, x_{2}, p, s, \lambda\right)\right| \\
& \quad \leq C \exp \left\{-a(\lambda+1)^{1 / 2}\left(\left|x_{1}-p\right|+\left|x_{2}-s\right|\right)\right\}\left(\left|x_{1}-p\right|+\left|x_{2}-s\right|\right)^{-1}
\end{aligned}
$$

hold. Here $a=a(\sigma)$.

Next, to formulate our result, we need to introduce the Hölder space $C^{\beta}=C^{\beta}\left(\mathbb{R}_{+}^{2}\right)$ of all continuous bounded functions $\varphi$ defined on $\mathbb{R}_{+}^{2}$ satisfying a Hölder condition with the indicator $\beta \in[0,1]$ with the norm

$$
\|f\|_{C^{\beta}\left(\mathbb{R}_{+}^{2}\right)}=\|f\|_{C\left(\mathbb{R}_{+}^{2}\right)}+\sup _{\substack{\left(x_{1}, x_{2}\right),\left(x_{1}^{\prime}, x_{2}^{\prime}\right) \in \mathbb{R}_{+}^{2} \\\left(x_{1}, x_{2}\right) \neq\left(x_{1}^{\prime}, x_{2}^{\prime}\right)}} \frac{\left|f\left(x_{1}, x_{2}\right)-f\left(x_{1}^{\prime}, x_{2}^{\prime}\right)\right|}{\left(\sqrt{\left|x_{1}-x_{1}^{\prime}\right|^{2}+\left|x_{2}-x_{2}^{\prime}\right|^{2}}\right)^{\beta}} .
$$

Here, $C\left(\mathbb{R}_{+}^{2}\right)$ denotes the Banach space of all continuous bounded functions $\varphi$ defined on $\mathbb{R}_{+}^{2}$ with the norm

$$
\|\varphi\|_{C\left(\mathbb{R}_{+}^{2}\right)}=\sup _{\left(x_{1}, x_{2}\right) \in \mathbb{R}_{+}^{2}}\left|\varphi\left(x_{1}, x_{2}\right)\right| .
$$

Clearly, from estimates (3) and (4) it follows that $A$ is a positive operator in $C\left(\mathbb{R}_{+}^{2}\right)$. Namely, we have the following.

Theorem 2 Let $\lambda \geq 0$. Then the following estimate

$$
\left\|(A+\lambda)^{-1}\right\|_{C\left(\mathbb{R}_{+}^{2}\right) \rightarrow C\left(\mathbb{R}_{+}^{2}\right)} \leq \frac{M}{1+\lambda}
$$

is valid.

Here, the structure of fractional spaces generated by the operator $A$ is investigated. The positivity of $A$ in Hölder spaces is studied. The organization of the present paper is as follows. In Section 2, the positivity of $A$ in Hölder spaces is established. In Section 3, the main theorem on the structure of fractional spaces $E_{\alpha}\left(A, C^{\beta}\left(\mathbb{R}_{+}^{2}\right)\right)$ generated by $A$ is investigated. In Section 4, applications on theorems on well-posedness in a Hölder space of parabolic and elliptic problems are presented. Finally, the conclusion is given.

2 Positivity of $A$ in Hölder spaces $C^{\beta}\left(\mathbb{R}_{+}^{2}\right)$

Theorem 3 Let $\beta \in(0,1)$. For $\lambda \geq 0$, we have the following estimate:

$$
\left\|(A+\lambda)^{-1}\right\|_{C^{\beta}\left(\mathbb{R}_{+}^{2}\right) \rightarrow C^{\beta}\left(\mathbb{R}_{+}^{2}\right)} \leq \frac{M(a)}{1+\lambda} .
$$


Proof Applying formula (2), the triangle inequality, the definition of $C^{\beta}$-norm, estimate (3), and Hilbert's inequality, we get

$$
\begin{aligned}
\left|(A+\lambda)^{-1} f\left(x_{1}, x_{2}\right)\right| & \\
\leq & \int_{0}^{\infty} \int_{-\infty}^{\infty}\left|G\left(x_{1}, x_{2}, p, s, \lambda\right)\right| f(p, s) \mid d s d p \\
\leq & M\|f\|_{C^{\beta}} \int_{0}^{\infty} \int_{-\infty}^{\infty} e^{-a(1+\lambda)^{1 / 2}\left(\left|x_{1}-p\right|+\left|x_{2}-s\right|\right)} \\
& \times\left(1+\ln \left\{1+\left((1+\lambda)^{1 / 2}\left(\left|x_{1}-p\right|+\left|x_{2}-s\right|\right)\right)^{-1}\right\}\right) d s d p \\
\leq & M\|f\|_{C^{\beta}}\left[\int_{0}^{\infty} \int_{-\infty}^{\infty} e^{-a(1+\lambda)^{1 / 2}\left(\left|x_{1}-p\right|+\left|x_{2}-s\right|\right)} d s d p\right. \\
& \left.+\int_{0}^{\infty} \int_{-\infty}^{\infty} e^{-a(1+\lambda)^{1 / 2}\left(\left|x_{1}-p\right|+\left|x_{2}-s\right|\right)}\left((1+\lambda)^{1 / 2}\left(\left|x_{1}-p\right|+\left|x_{2}-s\right|\right)\right)^{-1} d s d p\right] \\
\leq & \frac{M_{1}(a)}{1+\lambda}\|f\|_{C^{\beta}}+\frac{M_{2}(a)}{(1+\lambda)^{1 / 2}}\|f\|_{C^{\beta}} \int_{0}^{\infty} \int_{0}^{\infty} \frac{e^{-a(1+\lambda)^{1 / 2}(p+s)}}{p+s} d s d p \\
\leq & \frac{M_{3}(a)}{1+\lambda}\|f\|_{C^{\beta}} \text { for }\left(x_{1}, x_{2}\right) \in \mathbb{R}_{+}^{2} .
\end{aligned}
$$

Then from that it follows

$$
\sup _{\left(x_{1}, x_{2}\right) \in \mathbb{R}_{+}^{2}}\left|(A+\lambda)^{-1} f\left(x_{1}, x_{2}\right)\right| \leq \frac{M_{3}(a)}{1+\lambda}\|f\|_{C^{\beta}} .
$$

Without loss of generality, we can put $\tau, h>0$. Using formula (2) and the triangle inequality, we get

$$
\begin{aligned}
& \mid(A+\lambda)^{-1}\left[\frac{f\left(x_{1}+\tau, x_{2}+h\right)-f\left(x_{1}, x_{2}\right)}{\left(\tau^{2}+h^{2}\right)^{\beta / 2}}\right] \mid \\
& \leq \frac{1}{\left(\tau^{2}+h^{2}\right)^{\beta / 2}} \int_{0}^{\infty} \int_{-\infty}^{\infty}\left|G\left(x_{1}+\tau, x_{2}+h, p, s ; \lambda\right)-G\left(x_{1}, x_{2}, p, s, \lambda\right)\right| \\
& \quad \times|f(p, s)| d s d p
\end{aligned}
$$

for $\left(x_{1}, x_{2}\right),\left(x_{1}+\tau, x_{2}+h\right) \in \mathbb{R}_{+}^{2}$. Now, we will estimate the right-hand side of inequality (6). Let us consider two cases $\tau^{2}+h^{2}<1$ and $\tau^{2}+h^{2} \geq 1$ separately. First, we consider the case $\tau^{2}+h^{2}<1$. Using the triangle inequality, estimate (4), the definition of $C^{\beta}$-norm, Hilbert's inequality, and the Lagrange theorem, it follows that for some $x_{1}^{*}$ between $x_{1}, x_{1}+\tau$, and $x_{2}^{*}$ between $x_{2}, x_{2}+h$,

$$
\begin{aligned}
\left|(A+\lambda)^{-1}\left[\frac{f\left(x_{1}+\tau, x_{2}+h\right)-f\left(x_{1}, x_{2}\right)}{\left(\tau^{2}+h^{2}\right)^{\beta / 2}}\right]\right| \\
\leq M_{4}\|f\|_{C^{\beta}}\left[\frac{\tau}{\left(\tau^{2}+h^{2}\right)^{\beta / 2}} \int_{0}^{\infty} \int_{-\infty}^{\infty}\left|G_{x_{1}}\left(x_{1}^{*}, x_{2}^{*}, p, s ; \lambda\right)\right| d s d p\right. \\
\left.\quad+\frac{h}{\left(\tau^{2}+h^{2}\right)^{\beta / 2}} \int_{0}^{\infty} \int_{-\infty}^{\infty}\left|G_{x_{2}}\left(x_{1}^{*}, x_{2}^{*}, p, s ; \lambda\right)\right| d s d p\right] \\
\leq M_{4}\|f\|_{C^{\beta}}\left[\int_{0}^{\infty} \int_{-\infty}^{\infty}\left|G_{x_{1}}\left(x_{1}^{*}, x_{2}^{*}, p, s ; \lambda\right)\right| d s d p\right.
\end{aligned}
$$




$$
\begin{aligned}
& \left.+\int_{0}^{\infty} \int_{-\infty}^{\infty}\left|G_{x_{2}}\left(x_{1}^{*}, x_{2}^{*}, p, s ; \lambda\right)\right| d s d p\right] \\
\leq & M_{5}\|f\|_{C^{\beta}} \int_{0}^{\infty} \int_{-\infty}^{\infty} \frac{e^{-a(1+\lambda)^{1 / 2}\left(\left|x_{1}^{*}-p\right|+\left|x_{2}^{*}-s\right|\right)}}{\left|x_{1}^{*}-p\right|+\left|x_{2}^{*}-s\right|} d s d p \leq \frac{M_{6}(a)}{1+\lambda}\|f\|_{C^{\beta}} .
\end{aligned}
$$

Second, we consider the case $\tau^{2}+h^{2} \geq 1$. Using formula (2), the triangle inequality, estimate (3), the definition of $C^{\beta}$-norm, and estimate (6), we get

$$
\begin{aligned}
\mid(A+ & \lambda)^{-1}\left[\frac{f\left(x_{1}+\tau, x_{2}+h\right)-f\left(x_{1}, x_{2}\right)}{\left(\tau^{2}+h^{2}\right)^{\beta / 2}}\right] \mid \\
\leq & M_{7}\|f\|_{C^{\beta}}\left[\int_{0}^{\infty} \int_{-\infty}^{\infty} e^{-a(1+\lambda)^{1 / 2}\left(\left|x_{1}+\tau-p\right|+\left|x_{2}+h-s\right|\right)}\right. \\
& \times\left(1+\ln \left\{1+\left((\lambda+1)^{1 / 2}\left(\left|x_{1}+\tau-p\right|+\left|x_{2}+h-s\right|\right)\right)^{-1}\right\}\right) d s d p \\
& +\int_{0}^{\infty} \int_{-\infty}^{\infty} e^{-a(1+\lambda)^{1 / 2}\left(\left|x_{1}-p\right|+\left|x_{2}-s\right|\right)} \\
& \left.\times\left(1+\ln \left\{1+\left((\lambda+1)^{1 / 2}\left(\left|x_{1}-p\right|+\left|x_{2}-s\right|\right)\right)^{-1}\right\}\right) d s d p\right] \\
\leq & \frac{M_{8}(a)}{1+\lambda}\|f\|_{C^{\beta}} .
\end{aligned}
$$

Estimates (7) and (8) yield that

$$
\sup _{\substack{\left(x_{1}+\tau, x_{2}+h\right),\left(x_{1}, x_{2}\right) \in \mathbb{R}_{+}^{2} \\(\tau, h) \neq(0,0)}}\left|(A+\lambda)^{-1}\left[\frac{f\left(x_{1}+\tau, x_{2}+h\right)-f\left(x_{1}, x_{2}\right)}{\left(\tau^{2}+h^{2}\right)^{\beta / 2}}\right]\right| \leq \frac{M_{8}(a)}{1+\lambda}\|f\|_{C^{\beta}} .
$$

Combining estimates (5) and (9), we obtain

$$
\left\|(A+\lambda)^{-1}\right\|_{C^{\beta}\left(\mathbb{R}_{+}^{2}\right) \rightarrow C^{\beta}\left(\mathbb{R}_{+}^{2}\right)} \leq \frac{M(a)}{1+\lambda} .
$$

This finishes the proof of Theorem 3.

Note that from the commutativity of $A$ and its resolvent $(A+\lambda)^{-1}$, and Theorem 3 , we have the following theorem.

Theorem 4 Let $\lambda \geq 0$. Then the following estimate holds:

$$
\left\|(A+\lambda)^{-1}\right\|_{E_{\alpha}\left(A, C^{\beta}\left(\mathbb{R}_{+}^{2}\right)\right) \rightarrow E_{\alpha}\left(A, C^{\beta}\left(\mathbb{R}_{+}^{2}\right)\right)} \leq \frac{M(a)}{1+\lambda} .
$$

\section{The structure of fractional spaces $E_{\alpha}\left(A, C^{\beta}\left(\mathbb{R}_{+}^{2}\right)\right)$}

Suppose $\beta, 2 \alpha+\beta \in(0,1)$. Consider the fractional space $E_{\alpha}\left(A, C^{\beta}\left(\mathbb{R}_{+}^{2}\right)\right)$ and the Hölder space $C^{2 \alpha+\beta}\left(\mathbb{R}_{+}^{2}\right)$. In this section, we prove the following structure theorem.

Theorem 5 The norms of the spaces $E_{\alpha}\left(A, C^{\beta}\left(\mathbb{R}_{+}^{2}\right)\right)$ and $C^{2 \alpha+\beta}\left(\mathbb{R}_{+}^{2}\right)$ are equivalent. 
Proof Assume that $f \in C^{2 \alpha+\beta}\left(\mathbb{R}_{+}^{2}\right)$. Let $\left(x_{1}, x_{2}\right) \in \mathbb{R}_{+}^{2}$ and $\lambda>0$ be fixed. From formula (2) it follows that

$$
\begin{aligned}
& A(A+\lambda)^{-1} f\left(x_{1}, x_{2}\right) \\
& \quad=\frac{1}{\lambda+1} f\left(x_{1}, x_{2}\right)+\lambda \int_{0}^{\infty} \int_{-\infty}^{\infty} G\left(x_{1}, x_{2}, p, s, \lambda\right)\left(f\left(x_{1}, x_{2}\right)-f(p, s)\right) d s d p .
\end{aligned}
$$

Using equation (10), the triangle inequality, the following inequalities

$$
\begin{aligned}
& (a+b)^{p} \leq 2^{p}\left(a^{p}+b^{p}\right), \quad 0<p<1, a, b \geq 0 \\
& (a t)^{\theta} e^{-a t} \leq M, \quad a>0, \theta \in[0,1]
\end{aligned}
$$

estimates (10), (11), (12), and the definition of $C^{2 \alpha+\beta}$-norm, we obtain

$$
\begin{aligned}
\left|\lambda^{\alpha} A(A+\lambda)^{-1} f\left(x_{1}, x_{2}\right)\right| & \\
\leq & \frac{\lambda^{\alpha}}{\lambda+1}\left|f\left(x_{1}, x_{2}\right)\right|+\lambda^{\alpha+1} \int_{0}^{\infty} \int_{-\infty}^{\infty}\left|G\left(x_{1}, x_{2}, p, s, \lambda\right)\right|\left|f\left(x_{1}, x_{2}\right)-f(p, s)\right| d s d p \\
\leq & M_{9}\|f\|_{C^{2 \alpha+\beta}}\left[\frac{\lambda^{\alpha}}{\lambda+1}+\lambda^{\alpha+1} \int_{0}^{\infty} \int_{-\infty}^{\infty}\left|G\left(x_{1}, x_{2}, p, s, \lambda\right)\right|\right. \\
& \left.\times\left(\left|x_{1}-p\right|^{2}+\left|x_{2}-s\right|^{2}\right)^{\alpha+\beta / 2} d s d p\right] \\
\leq & M_{10}\|f\|_{C^{2 \alpha+\beta}}\left[\frac{\lambda^{\alpha}}{\lambda+1}+\lambda^{\alpha+1} \int_{0}^{\infty} \int_{-\infty}^{\infty} e^{-a(1+\lambda)^{1 / 2}\left(\left|x_{1}-p\right|+\left|x_{2}-s\right|\right)}\right. \\
& \left.\times\left(1+\ln \left\{1+\left((1+\lambda)^{1 / 2}\left(\left|x_{1}-p\right|+\left|x_{2}-s\right|\right)\right)^{-1}\right\}\right)\left(\left|x_{1}-p\right|^{2}+\left|x_{2}-s\right|^{2}\right)^{\alpha+\beta / 2} d s d p\right] \\
\leq & M_{11}(a)\|f\|_{C^{2 \alpha+\beta}}\left[\frac{\lambda^{\alpha}}{\lambda+1}+\frac{\lambda^{\alpha+1}}{(1+\lambda)^{1+\alpha+\beta / 2}}\right] \leq M_{12}(a)\|f\|_{C^{2 \alpha+\beta}} \\
\quad & \text { for }\left(x_{1}, x_{2}\right) \in \mathbb{R}_{+}^{2} .
\end{aligned}
$$

Thus, it follows from estimate (13) that

$$
\sup _{\lambda>0} \sup _{\left(x_{1}, x_{2}\right) \in \mathbb{R}_{+}^{2}}\left|\lambda^{\alpha} A(A+\lambda)^{-1} f\left(x_{1}, x_{2}\right)\right| \leq M_{12}(a)\|f\|_{C^{2 \alpha+\beta}} .
$$

Let $\lambda>0$ and $\left(x_{1}, x_{2}\right) \in \mathbb{R}_{+}^{2}$ be fixed. Using equation (10), we can write

$$
\begin{aligned}
\lambda^{\alpha} A(A+\lambda)^{-1}\left[\frac{f\left(x_{1}+\tau, x_{2}+h\right)-f\left(x_{1}, x_{2}\right)}{\left(\tau^{2}+h^{2}\right)^{\beta / 2}}\right] \\
=\frac{\lambda^{\alpha}}{\lambda+1}\left[\frac{f\left(x_{1}+\tau, x_{2}+h\right)-f\left(x_{1}, x_{2}\right)}{\left(\tau^{2}+h^{2}\right)^{\beta / 2}}\right] \\
\quad+\lambda^{\alpha+1} \int_{0}^{\infty} \int_{-\infty}^{\infty} G\left(x_{1}+\tau, x_{2}+h, p, s ; \lambda\right)\left[\frac{f\left(x_{1}+\tau, x_{2}+h\right)-f(p, s)}{\left(\tau^{2}+h^{2}\right)^{\beta / 2}}\right] d s d p \\
\quad-\lambda^{\alpha+1} \int_{0}^{\infty} \int_{-\infty}^{\infty} G\left(x_{1}, x_{2}, p, s ; \lambda\right)\left[\frac{f\left(x_{1}, x_{2}\right)-f(p, s)}{\left(\tau^{2}+h^{2}\right)^{\beta / 2}}\right] d s d p .
\end{aligned}
$$


Now, we will estimate the right-hand side of equation (15). We consider two cases $\tau^{2}+$ $h^{2}<1$ and $\tau^{2}+h^{2} \geq 1$, respectively. Let us first assume that $\tau^{2}+h^{2}<1$. Furthermore, this situation will be considered in two cases: $(1+\lambda)\left(\tau^{2}+h^{2}\right) \geq 1$ and $(1+\lambda)\left(\tau^{2}+h^{2}\right) \leq 1$. Let $(1+\lambda)\left(\tau^{2}+h^{2}\right) \geq 1$. From equation (15), the triangle inequality, the definition of $C^{2 \alpha+\beta}$ norm, the assumptions $\tau^{2}+h^{2}<1$ and $(1+\lambda)\left(\tau^{2}+h^{2}\right) \geq 1$, it follows that

$$
\begin{aligned}
\left|\lambda^{\alpha} A(A+\lambda)^{-1}\left[\frac{f\left(x_{1}+\tau, x_{2}+h\right)-f\left(x_{1}, x_{2}\right)}{\left(\tau^{2}+h^{2}\right)^{\beta / 2}}\right]\right| \\
\leq M_{13}\|f\|_{C^{2 \alpha+\beta}}\left[\frac{\lambda^{\alpha}}{(1+\lambda)\left(\tau^{2}+h^{2}\right)^{\beta / 2}}\right. \\
\quad+\frac{\lambda^{\alpha+1}}{\left(\tau^{2}+h^{2}\right)^{\beta / 2}} \int_{0}^{\infty} \int_{-\infty}^{\infty}\left|G\left(x_{1}+\tau, x_{2}+h, p, s ; \lambda\right)\right| \\
\quad \times\left(\left|x_{1}+\tau-p\right|^{2}+\left|x_{2}+h-s\right|^{2}\right)^{\alpha+\beta / 2} d s d p \\
\left.\quad+\frac{\lambda^{\alpha+1}}{\left(\tau^{2}+h^{2}\right)^{\beta / 2}} \int_{0}^{\infty} \int_{-\infty}^{\infty}\left|G\left(x_{1}, x_{2}, p, s ; \lambda\right)\right|\left(\left|x_{1}-p\right|^{2}+\left|x_{2}-s\right|^{2}\right)^{\alpha+\beta / 2} d s d p\right] \\
=M_{13}\|f\|_{C^{2 \alpha+\beta}\left[J_{1}+J_{2}+J_{3}\right] .}
\end{aligned}
$$

We will estimate $J_{i}, i=1,2,3$, separately.

First, let us estimate $J_{1}$. Clearly, by the assumption $(1+\lambda)\left(\tau^{2}+h^{2}\right) \geq 1$, we have

$$
J_{1} \leq 1
$$

From estimates (3), (11), (12), and the assumption $(1+\lambda)\left(\tau^{2}+h^{2}\right) \geq 1$, it follows that

$$
\begin{aligned}
J_{2} \leq & M_{14} \frac{\lambda^{\alpha+1}}{\left(\tau^{2}+h^{2}\right)^{\beta / 2}} \int_{0}^{\infty} \int_{-\infty}^{\infty} e^{-a(1+\lambda)^{1 / 2}\left(\left|x_{1}+\tau-p\right|+\left|x_{2}+h-s\right|\right)} \\
& \times\left(1+\ln \left\{1+\left((\lambda+1)^{1 / 2}\left(\left|x_{1}+\tau-p\right|+\left|x_{2}+h-s\right|\right)\right)^{-1}\right\}\right) \\
& \times\left(\left|x_{1}+\tau-p\right|^{2}+\left|x_{2}+h-s\right|^{2}\right)^{\alpha+\beta / 2} d s d p \\
\leq & M_{15}(a) \frac{\lambda^{\alpha+1}}{(1+\lambda)^{1+\alpha+\beta / 2}\left(\tau^{2}+h^{2}\right)^{\beta / 2}} \leq M_{15}(a) .
\end{aligned}
$$

Estimates (3), (11), (12), and the assumption $(1+\lambda)\left(\tau^{2}+h^{2}\right) \geq 1$ yield that

$$
\begin{aligned}
J_{3} \leq & M_{16} \frac{\lambda^{\alpha+1}}{\left(\tau^{2}+h^{2}\right)^{\beta / 2}} \int_{0}^{\infty} \int_{-\infty}^{\infty} e^{-a(1+\lambda)^{1 / 2}\left(\left|x_{1}-p\right|+\left|x_{2}-s\right|\right)} \\
& \times\left(1+\ln \left\{1+\left((\lambda+1)^{1 / 2}\left(\left|x_{1}-p\right|+\left|x_{2}-s\right|\right)\right)^{-1}\right\}\right)\left(\left|x_{1}-p\right|^{2}+\left|x_{2}-s\right|^{2}\right)^{\alpha+\beta / 2} d s d p \\
\leq & \frac{M_{17}(a) \lambda^{\alpha+1}}{(1+\lambda)^{1+\alpha+\beta / 2}\left(\tau^{2}+h^{2}\right)^{\beta / 2}} \leq M_{17}(a) .
\end{aligned}
$$

Combining estimates (17)-(19), we get

$$
\left|\lambda^{\alpha} A(A+\lambda)^{-1}\left[\frac{f\left(x_{1}+\tau, x_{2}+h\right)-f\left(x_{1}, x_{2}\right)}{\left(\tau^{2}+h^{2}\right)^{\beta / 2}}\right]\right| \leq M_{18}(a)\|f\|_{C^{2 \alpha+\beta}} .
$$


Now, let us consider the case $(1+\lambda)\left(\tau^{2}+h^{2}\right) \leq 1$. Then, using equation (10), we can write

$$
\begin{aligned}
\lambda^{\alpha} A(A+\lambda)^{-1}\left[\frac{f\left(x_{1}+\tau, x_{2}+h\right)-f\left(x_{1}, x_{2}\right)}{\left(\tau^{2}+h^{2}\right)^{\beta / 2}}\right] & \lambda^{\alpha}\left[\frac{f\left(x_{1}+\tau, x_{2}+h\right)-f\left(x_{1}, x_{2}\right)}{\left(\tau^{2}+h^{2}\right)^{\beta / 2}}\right] \\
= & +\lambda^{\alpha+1} \int_{0}^{\infty} \int_{-\infty}^{\infty} G\left(x_{1}+\tau, x_{2}+h, p, s ; \lambda\right)\left[\frac{f\left(x_{1}+\tau, x_{2}+h\right)-f\left(x_{1}, x_{2}\right)}{\left(\tau^{2}+h^{2}\right)^{\beta / 2}}\right] d s d p \\
& +\lambda^{\alpha+1} \int_{0}^{\infty} \int_{-\infty}^{\infty}\left[G\left(x_{1}+\tau, x_{2}+h, p, s ; \lambda\right)-G\left(x_{1}, x_{2}, p, s ; \lambda\right)\right] \\
& \times\left[\frac{f\left(x_{1}, x_{2}\right)-f(p, s)}{\left(\tau^{2}+h^{2}\right)^{\beta / 2}}\right] d s d p .
\end{aligned}
$$

From equation (21), the triangle inequality, the Lagrange theorem, the definition of $C^{2 \alpha+\beta}$ norm, and the assumption $\tau^{2}+h^{2}<1$, it follows that for some $x_{1}^{*}$ between $x_{1}, x_{2}+\tau$, and $x_{2}^{*}$ between $x_{2}, x_{2}+h$,

$$
\begin{aligned}
&\left|\lambda^{\alpha} A(A+\lambda)^{-1}\left[\frac{f\left(x_{1}+\tau, x_{2}+h\right)-f\left(x_{1}, x_{2}\right)}{\left(\tau^{2}+h^{2}\right)^{\beta / 2}}\right]\right| \\
& \leq M_{19}\|f\|_{C^{2 \alpha+\beta}}\left[\frac{\lambda^{\alpha}}{\lambda+1}\left(\tau^{2}+h^{2}\right)^{\alpha}+\lambda^{\alpha+1}\left(\tau^{2}+h^{2}\right)^{\alpha}\right. \\
& \times \int_{0}^{\infty} \int_{-\infty}^{\infty}\left|G\left(x_{1}+\tau, x_{2}+h, p, s ; \lambda\right)\right| d s d p \\
&+\frac{\lambda^{\alpha+1} \tau}{\left(\tau^{2}+h^{2}\right)^{\beta / 2}} \int_{0}^{\infty} \int_{-\infty}^{\infty}\left|G_{x_{1}}\left(x_{1}^{*}, x_{2}^{*}, p, s ; \lambda\right)\right|\left(\left|x_{1}-p\right|^{2}+\left|x_{2}-s\right|^{2}\right)^{\alpha+\beta / 2} d s d p \\
&\left.+\frac{\lambda^{\alpha+1} h}{\left(\tau^{2}+h^{2}\right)^{\beta / 2}} \int_{0}^{\infty} \int_{-\infty}^{\infty}\left|G_{x_{2}}\left(x_{1}^{*}, x_{2}^{*}, p, s ; \lambda\right)\right|\left(\left|x_{1}-p\right|^{2}+\left|x_{2}-s\right|^{2}\right)^{\alpha+\beta / 2} d s d p\right] \\
&= M_{19}\|f\|_{C^{2 \alpha+\beta}}\left[L_{1}+L_{2}+L_{3}+L_{4}\right] .
\end{aligned}
$$

We will estimate $L_{i}, i=1, \ldots, 4$, separately. Let us start with $L_{1}$. Clearly, we have

$$
L_{1} \leq 1
$$

Using estimates (3), (4), (11), (12), we obtain

$$
\begin{aligned}
L_{2} \leq & M_{20} \lambda \int_{0}^{\infty} \int_{-\infty}^{\infty} e^{-a(1+\lambda)^{1 / 2}\left(\left|x_{1}+\tau-p\right|+\left|x_{2}+h-s\right|\right)} \\
& \times\left(1+\ln \left\{1+\left((\lambda+1)^{1 / 2}\left(\left|x_{1}+\tau-p\right|+\left|x_{2}+h-s\right|\right)\right)^{-1}\right\}\right) d s d p \leq M .
\end{aligned}
$$

Note that by the triangle inequality and the fact that $x_{1}^{*}$ between $x_{1}, x_{1}+\tau$, we get

$$
\begin{aligned}
& \left|x_{1}^{*}-p\right| \geq\left|x_{1}-p\right|-\left|x_{1}^{*}-x_{1}\right| \geq\left|x_{1}-p\right|-\tau, \\
& \left|x_{2}^{*}-s\right| \geq\left|x_{2}-s\right|-\left|x_{2}^{*}-x_{2}\right| \geq\left|x_{2}-s\right|-h .
\end{aligned}
$$


By inequalities (11), (12), estimates (4), (24), (25), and Hilbert's inequality, we have

$$
\begin{aligned}
L_{3} \leq & M_{21} \frac{\lambda^{\alpha+1} \tau}{\left(\tau^{2}+h^{2}\right)^{\beta / 2}} \int_{0}^{\infty} \int_{-\infty}^{\infty} \frac{e^{-a(1+\lambda)^{1 / 2}\left(\left|x_{1}^{*}-p\right|+\left|x_{2}^{*}-s\right|\right)}}{\left|x_{1}^{*}-p\right|+\left|x_{2}^{*}-s\right|}\left(\left|x_{1}-p\right|+\left|x_{2}-s\right|\right)^{2 \alpha+\beta} d s d p \\
\leq & M_{22} \frac{\lambda^{\alpha+1} \tau}{\left(\tau^{2}+h^{2}\right)^{\beta / 2}} \int_{0}^{\infty} \int_{-\infty}^{\infty} e^{-a(1+\lambda)^{1 / 2}\left(\left|x_{1}^{*}-p\right|+\left|x_{2}^{*}-s\right|\right)}\left(\left|x_{1}^{*}-p\right|+\left|x_{2}^{*}-s\right|\right)^{2 \alpha+\beta-1} d s d p \\
& +M_{22} \frac{\lambda^{\alpha+1} \tau}{\left(\tau^{2}+h^{2}\right)^{\beta / 2}} \int_{0}^{\infty} \int_{-\infty}^{\infty} e^{-a(1+\lambda)^{1 / 2}\left(\left|x_{1}^{*}-p\right|+\left|x_{2}^{*}-s\right|\right)} \\
& \times\left(\left|x_{1}^{*}-p\right|+\left|x_{2}^{*}-s\right|\right)^{-1}(\tau+h)^{2 \alpha+\beta} d s d p \\
\leq & M_{23}(a)\left[\frac{\lambda^{\alpha+1} \tau}{(1+\lambda)^{1+\alpha+\beta / 2}\left(\tau^{2}+h^{2}\right)^{\beta / 2}}+\frac{\lambda^{\alpha+1} \tau(\tau+h)^{2 \alpha+\beta}}{(1+\lambda)\left(\tau^{2}+h^{2}\right)^{\beta / 2}}\right] \leq M_{24}(a) .
\end{aligned}
$$

Similarly, we get

$$
L_{4} \leq M_{24}(a)
$$

Combining estimates (22), (23), (26), (27), we obtain that for $\tau^{2}+h^{2}<1,(1+\lambda)^{1 / 2}\left(\tau^{2}+\right.$ $\left.h^{2}\right) \leq 1$,

$$
\left|\lambda^{\alpha} A(A+\lambda)^{-1}\left[\frac{f\left(x_{1}+\tau, x_{2}+h\right)-f\left(x_{1}, x_{2}\right)}{\left(\tau^{2}+h^{2}\right)^{\beta / 2}}\right]\right| \leq M_{25}(a)\|f\|_{C^{2 \alpha+\beta}} .
$$

It follows from estimates (20) and (28) that for $\tau^{2}+h^{2}<1, \lambda>0$,

$$
\left|\lambda^{\alpha} A(A+\lambda)^{-1}\left[\frac{f\left(x_{1}+\tau, x_{2}+h\right)-f\left(x_{1}, x_{2}\right)}{\left(\tau^{2}+h^{2}\right)^{\beta / 2}}\right]\right| \leq M_{26}(a)\|f\|_{C^{2 \alpha+\beta}} .
$$

Next, let us assume that $\tau^{2}+h^{2} \geq 1$. By equation (10), we have

$$
\begin{aligned}
\lambda^{\alpha} A(A+\lambda)^{-1}\left[\frac{f\left(x_{1}+\tau, x_{2}+h\right)-f\left(x_{1}, x_{2}\right)}{\left(\tau^{2}+h^{2}\right)^{\beta / 2}}\right] \\
=\frac{\lambda^{\alpha}}{\lambda+1}\left[\frac{f\left(x_{1}+\tau, x_{2}+h\right)-f\left(x_{1}, x_{2}\right)}{\left(\tau^{2}+h^{2}\right)^{\beta / 2}}\right] \\
\quad+\lambda^{\alpha+1} \int_{0}^{\infty} \int_{-\infty}^{\infty} G\left(x_{1}+\tau, x_{2}+h, p, s ; \lambda\right)\left[\frac{f\left(x_{1}+\tau, x_{2}+h\right)-f(p, s)}{\left(\tau^{2}+h^{2}\right)^{\beta / 2}}\right] d s d p \\
\quad-\lambda^{\alpha+1} \int_{0}^{\infty} \int_{-\infty}^{\infty} G\left(x_{1}, x_{2}, p, s ; \lambda\right)\left[\frac{f\left(x_{1}, x_{2}\right)-f(p, s)}{\left(\tau^{2}+h^{2}\right)^{\beta / 2}}\right] d s d p .
\end{aligned}
$$

Equation (30), estimates (3), (11), Hilbert's inequality, the triangle inequality, the definition of $C^{2 \alpha+\beta}$-norm, and the assumption $\tau^{2}+h^{2} \geq 1$ yield that for $\lambda>0$,

$$
\begin{aligned}
& \left|\lambda^{\alpha} A(A+\lambda)^{-1}\left[\frac{f\left(x_{1}+\tau, x_{2}+h\right)-f\left(x_{1}, x_{2}\right)}{\left(\tau^{2}+h^{2}\right)^{\beta / 2}}\right]\right| \\
& \leq M_{27}\|f\|_{C^{2 \alpha+\beta}}\left[1+\lambda^{\alpha+1} \int_{0}^{\infty} \int_{-\infty}^{\infty}\left|G\left(x_{1}+\tau, x_{2}+h, p, s ; \lambda\right)\right|\right. \\
& \quad \times\left(\left|x_{1}+\tau-p\right|^{2}+\left|x_{2}+h-s\right|^{2}\right)^{\alpha+\beta / 2} d s d p
\end{aligned}
$$




$$
\begin{aligned}
& +\lambda^{\alpha+1} \int_{0}^{\infty} \int_{-\infty}^{\infty}\left|G\left(x_{1}, x_{2}, p, s ; \lambda\right)\right|\left(\left|x_{1}-p\right|^{2}+\left|x_{2}-s\right|^{2}\right)^{\alpha+\beta / 2} d s d p \\
\leq & M_{28}(a)\|f\|_{C^{2 \alpha+\beta}}\left[1+\frac{\lambda^{\alpha+1}}{(1+\lambda)^{1+\alpha+\beta / 2}}+\frac{\lambda^{\alpha+1}}{(1+\lambda)^{1+\alpha+\beta / 2}}\right] \\
\leq & M_{29}(a)\|f\|_{C^{2 \alpha+\beta}} .
\end{aligned}
$$

From estimates (29) and (31) it follows that

$$
\begin{aligned}
& \sup _{\lambda>0} \sup _{\substack{\left(x_{1}+\tau, x_{2}+h\right),\left(x_{1}, x_{2}\right) \in \mathbb{R}_{+}^{2} \\
(\tau, h) \neq(0,0)}}\left|\lambda^{\alpha} A(A+\lambda)^{-1}\left[\frac{f\left(x_{1}+\tau, x_{2}+h\right)-f\left(x_{1}, x_{2}\right)}{\left(\tau^{2}+h^{2}\right)^{\beta / 2}}\right]\right| \\
& \leq M_{30}(a)\|f\|_{C^{2 \alpha+\beta}} .
\end{aligned}
$$

Combining estimates (14) and (32), we obtain

$$
C^{2 \alpha+\beta}\left(\mathbb{R}_{+}^{2}\right) \subset E_{\alpha}\left(A, C^{\beta}\left(\mathbb{R}_{+}^{2}\right)\right)
$$

Now, we will prove that $E_{\alpha}=E_{\alpha}\left(A, C^{\beta}\left(\mathbb{R}_{+}^{2}\right)\right) \subset C^{2 \alpha+\beta}\left(\mathbb{R}_{+}^{2}\right)$. By Theorem $4, A$ is a positive operator in the Banach space $E_{\alpha}$. Hence, for $V \in E_{\alpha}$, we have

$$
V=\int_{0}^{\infty} A(\lambda+A)^{-2} V d \lambda
$$

Let $f \in E_{\alpha}$. It follows from formula (2) and equation (33) that

$$
\begin{aligned}
f\left(x_{1}, x_{2}\right) & =\int_{0}^{\infty} A(\lambda+A)^{-2} f\left(x_{1}, x_{2}\right) d \lambda \\
& =\int_{0}^{\infty} \int_{0}^{\infty} \int_{-\infty}^{\infty} G\left(x_{1}, x_{2}, p, s ; \lambda\right) A(\lambda+A)^{-1} f(p, s) d s d p d \lambda .
\end{aligned}
$$

Using the triangle inequality, equation (34), estimate (3), and the definition of $E_{\alpha}$-norm, we obtain

$$
\begin{aligned}
\left|f\left(x_{1}, x_{2}\right)\right| \leq & M_{31}\|f\|_{E_{\alpha}} \int_{0}^{\infty} \int_{0}^{\infty} \int_{-\infty}^{\infty} \frac{1}{\lambda^{\alpha}} e^{-a(1+\lambda)^{1 / 2}\left(\left|x_{1}-p\right|+\left|x_{2}-s\right|\right)} \\
& \times\left(1+\ln \left\{1+\left((1+\lambda)^{1 / 2}\left(\left|x_{1}-p\right|+\left|x_{2}-s\right|\right)\right)^{-1}\right\}\right) d s d p d \lambda \\
\leq & M_{32}(a)\|f\|_{E_{\alpha}} \int_{0}^{\infty} \frac{1}{\lambda^{\alpha}(1+\lambda)} d \lambda \leq \frac{M_{33}(a)}{\alpha(1-\alpha)}\|f\|_{E_{\alpha} .}
\end{aligned}
$$

Thus,

$$
\sup _{\left(x_{1}, x_{2}\right) \in \mathbb{R}_{+}^{2}}\left|f\left(x_{1}, x_{2}\right)\right| \leq \frac{M_{33}(a)}{\alpha(1-\alpha)}\|f\|_{E_{\alpha}} .
$$

Let $\left(x_{1}, x_{2}\right) \in \mathbb{R}_{+}^{2}$ be fixed. From equation (34) it follows that

$$
\begin{aligned}
& \frac{f\left(x_{1}+\tau, x_{2}+h\right)-f\left(x_{1}, x_{2}\right)}{\left(\tau^{2}+h^{2}\right)^{\alpha+\beta / 2}} \\
& \quad=\int_{0}^{\infty} \int_{0}^{\infty} \int_{-\infty}^{\infty} \frac{G\left(x_{1}+\tau, x_{2}+h, p, s ; \lambda\right)}{\left(\tau^{2}+h^{2}\right)^{\alpha+\beta / 2} \lambda^{\alpha}} \lambda^{\alpha} A(A+\lambda)^{-1}
\end{aligned}
$$




$$
\begin{aligned}
& \times\left(f(p, s)-f\left(x_{1}, x_{2}\right)\right) d s d p d \lambda \\
& -\int_{0}^{\infty} \int_{0}^{\infty} \int_{-\infty}^{\infty} \frac{G\left(x_{1}, x_{2}, p, s ; \lambda\right)}{\left(\tau^{2}+h^{2}\right)^{\alpha+\beta / 2} \lambda^{\alpha}} \lambda^{\alpha} A(A+\lambda)^{-1}\left(f(p, s)-f\left(x_{1}, x_{2}\right)\right) d s d p d \lambda .
\end{aligned}
$$

Now, we will estimate the right-hand side of equation (36). We consider two cases $\tau^{2}+h^{2}<$ 1 and $\tau^{2}+h^{2} \geq 1$. Let us first assume that $\tau^{2}+h^{2}<1$. By equation (36), the triangle inequality, the Lagrange theorem, the definition of $E_{\alpha}$-norm, and the assumption $\tau^{2}+h^{2}<1$, we have, for some $x_{1}^{*}$ between $x_{1}, x_{1}+\tau$, and $x_{2}^{*}$ between $x_{2}, x_{2}+h$, that

$$
\begin{aligned}
\left|\frac{f\left(x_{1}+\tau, x_{2}+h\right)-f\left(x_{1}, x_{2}\right)}{\left(\tau^{2}+h^{2}\right)^{\alpha+\beta / 2}}\right| & \\
\leq & M_{34} \mid f \|_{E_{\alpha}}\left[\int_{0}^{1} \int_{0}^{\infty} \int_{-\infty}^{\infty}\left[\frac{\left|G\left(x_{1}+\tau, x_{2}+h, p, s ; \lambda\right)-G\left(x_{1}, x_{2}, p, s ; \lambda\right)\right|}{\left(\tau^{2}+h^{2}\right)^{\alpha+\beta / 2} \lambda^{\alpha}}\right]\right. \\
\quad & \quad\left(\left|x_{1}-p\right|^{2}+\left|x_{2}-s\right|^{2}\right)^{\beta / 2} d s d p d \lambda \\
& +\int_{1}^{1 /\left(\tau^{2}+h^{2}\right)} \int_{0}^{\infty} \int_{-\infty}^{\infty}\left[\frac{\left|G\left(x_{1}+\tau, x_{2}+h, p, s ; \lambda\right)-G\left(x_{1}, x_{2}, p, s ; \lambda\right)\right|}{\left(\tau^{2}+h^{2}\right)^{\alpha+\beta / 2} \lambda^{\alpha}}\right] \\
& \times\left(\left|x_{1}-p\right|^{2}+\left|x_{2}-s\right|^{2}\right)^{\beta / 2} d s d p d \lambda \\
& +\int_{1 /\left(\tau^{2}+h^{2}\right)}^{\infty} \int_{0}^{\infty} \int_{-\infty}^{\infty}\left[\frac{\left|G\left(x_{1}+\tau, x_{2}+h, p, s ; \lambda\right)-G\left(x_{1}, x_{2}, p, s ; \lambda\right)\right|}{\left(\tau^{2}+h^{2}\right)^{\alpha+\beta / 2} \lambda^{\alpha}}\right] \\
& \left.\times\left(\left|x_{1}-p\right|^{2}+\left|x_{2}-s\right|^{2}\right)^{\beta / 2} d s d p d \lambda\right] \\
= & M_{34}\|f\|_{E_{\alpha}}\left[I_{1}+I_{2}+I_{3}\right] .
\end{aligned}
$$

We will estimate $I_{i}, i=1,2,3$, separately. Using the triangle inequality, estimates (4), (11), (25), (24), (12), the Lagrange theorem, and the assumption $\tau^{2}+h^{2}<1$, we obtain

$$
\begin{aligned}
I_{1} \leq & M_{35}\left[\int_{0}^{1} \int_{0}^{\infty} \int_{-\infty}^{\infty}\left[\frac{\tau\left|G_{x_{1}}\left(x_{1}^{*}, x_{2}^{*}, p, s ; \lambda\right)\right|+h\left|G_{x_{2}}\left(x_{1}^{*}, x_{2}^{*}, p, s ; \lambda\right)\right|}{\left(\tau^{2}+h^{2}\right)^{\alpha+\beta / 2} \lambda^{\alpha}}\right]\right. \\
& \left.\times\left(\left|x_{1}-p\right|^{2}+\left|x_{2}-s\right|^{2}\right)^{\beta / 2} d s d p d \lambda\right] \\
\leq & \int_{0}^{1} \int_{0}^{\infty} \int_{-\infty}^{\infty}\left[\frac{e^{-a(1+\lambda)^{1 / 2}\left(\left|x_{1}^{*}-p\right|+\left|x_{2}^{*}-s\right|\right)}}{\left(\left|x_{1}^{*}-p\right|+\left|x_{2}^{*}-s\right|\right) \lambda^{\alpha}}\right]\left(\left|x_{1}-p\right|^{2}+\left|x_{2}-s\right|^{2}\right)^{\beta / 2} d s d p d \lambda \\
\leq & M_{36}\left[\int_{0}^{1} \int_{0}^{\infty} \int_{-\infty}^{\infty} \frac{e^{-a(1+\lambda)^{1 / 2}\left(\left|x_{1}^{*}-p\right|+\left|x_{2}^{*}-s\right|\right)}\left(\left|x_{1}^{*}-p\right|+\left|x_{2}^{*}-s\right|\right)^{\beta}}{\lambda^{\alpha}\left(\left|x_{1}^{*}-p\right|+\left|x_{2}^{*}-s\right|\right)} d s d p d \lambda\right. \\
& \left.+\int_{0}^{1} \int_{0}^{\infty} \int_{-\infty}^{\infty} \frac{e^{-a(1+\lambda)^{1 / 2}\left(\left|x_{1}^{*}-p\right|+\left|x_{2}^{*}-s\right|\right)}(\tau+h)^{\beta}}{\lambda^{\alpha}\left(\left|x_{1}^{*}-p\right|+\left|x_{2}^{*}-s\right|\right)} d s d p d \lambda\right] \\
\leq & M_{37}(a)\left[\int_{0}^{1} \frac{1}{\lambda^{\alpha+\beta / 2+1 / 2}} d \lambda+\int_{0}^{1} \frac{1}{\lambda^{\alpha+1 / 2}} d \lambda\right] \\
\leq & M_{38}(a) .
\end{aligned}
$$


From the triangle inequality, estimates (4), (11), (12), (24), (25), the Lagrange theorem, and the assumption $\tau^{2}+h^{2}<1$, it follows that

$$
\begin{aligned}
I_{2} \leq & M_{39}\left[\int_{1}^{1 /\left(\tau^{2}+h^{2}\right)} \int_{0}^{\infty} \int_{-\infty}^{\infty}\left[\frac{\tau\left|G_{x_{1}}\left(x_{1}^{*}, x_{2}^{*}, p, s ; \lambda\right)\right|+h\left|G_{x_{2}}\left(x_{1}^{*}, x_{2}^{*}, p, s ; \lambda\right)\right|}{\left(\tau^{2}+h^{2}\right)^{\alpha+\beta / 2} \lambda^{\alpha}}\right]\right. \\
& \left.\times\left(\left|x_{1}-p\right|^{2}+\left|x_{2}-s\right|^{2}\right)^{\beta / 2} d s d p d \lambda\right] \\
\leq & M_{40}\left[\int_{1}^{1 /\left(\tau^{2}+h^{2}\right)} \int_{0}^{\infty} \int_{-\infty}^{\infty} \frac{(\tau+h) e^{-a(1+\lambda)^{1 / 2}\left(\left|x_{1}^{*}-p\right|+\left|x_{2}^{*}-s\right|\right)}\left(\left|x_{1}-p\right|+\left|x_{2}-s\right|\right)^{\beta}}{\left(\tau^{2}+h^{2}\right)^{\alpha+\beta / 2} \lambda^{\alpha}\left(\left|x_{1}^{*}-p\right|+\left|x_{2}^{*}-s\right|\right)} d s d p d \lambda\right] \\
\leq & M_{41}\left[\int_{1}^{1 /\left(\tau^{2}+h^{2}\right)} \int_{0}^{\infty} \int_{-\infty}^{\infty} \frac{(\tau+h) e^{-a(1+\lambda)^{1 / 2}\left(\left|x_{1}^{*}-p\right|+\left|x_{2}^{*}-s\right|\right)}\left(\left|x_{1}^{*}-p\right|+\left|x_{2}^{*}-s\right|\right)^{\beta}}{\left(\tau^{2}+h^{2}\right)^{\alpha+\beta / 2} \lambda^{\alpha}\left(\left|x_{1}^{*}-p\right|+\left|x_{2}^{*}-s\right|\right)} d s d p d \lambda\right. \\
& \left.+\int_{1}^{1 /\left(\tau^{2}+h^{2}\right)} \int_{0}^{\infty} \int_{-\infty}^{\infty} \frac{(\tau+h) e^{-a(1+\lambda)^{1 / 2}\left(\left|x_{1}^{*}-p\right|+\left|x_{2}^{*}-s\right|\right)}(\tau+h)^{\beta}}{\left(\tau^{2}+h^{2}\right)^{\alpha+\beta / 2} \lambda^{\alpha}\left(\left|x_{1}^{*}-p\right|+\left|x_{2}^{*}-s\right|\right)} d s d p d \lambda\right] \\
\leq & M_{42}(a)\left[\int_{1}^{1 /\left(\tau^{2}+h^{2}\right)} \frac{\left(\tau^{2}+h^{2}\right)^{1 / 2}}{\left(\tau^{2}+h^{2}\right)^{\alpha+\beta / 2} \lambda^{\alpha+\beta / 2+1 / 2}} d \lambda+\int_{1}^{1 /\left(\tau^{2}+h^{2}\right)} \frac{\left(\tau^{2}+h^{2}\right)^{1 / 2+\beta / 2}}{\left(\tau^{2}+h^{2}\right)^{\alpha+\beta / 2} \lambda^{\alpha+1 / 2}} d \lambda\right] \\
\leq & M_{43}(a) .
\end{aligned}
$$

Using the triangle inequality, estimates (3), (24), (25), (12), (11), the assumption $\tau^{2}+h^{2}<1$, and the following estimate

$$
\begin{aligned}
& \left|x_{1}-p\right|=\left|x_{1}-p+\tau-\tau\right| \leq\left|x_{1}-p+\tau\right|+\tau, \\
& \left|x_{2}-s\right|=\left|x_{2}-s+h-h\right| \leq\left|x_{2}-s+h\right|+h,
\end{aligned}
$$

we obtain

$$
\begin{aligned}
I_{3} \leq & M_{44}\left[\int_{1 /\left(\tau^{2}+h^{2}\right)}^{\infty} \int_{0}^{\infty} \int_{-\infty}^{\infty} \frac{e^{-a(1+\lambda)^{1 / 2}\left(\left|x_{1}+\tau-p\right|+\left|x_{2}+h-s\right|\right)}}{\left(\tau^{2}+h^{2}\right)^{\alpha+\beta / 2} \lambda^{\alpha}}\right. \\
& \times\left(1+\ln \left\{1+\left((\lambda+1)^{1 / 2}\left(\left|x_{1}+\tau-p\right|+\left|x_{2}+h-s\right|\right)\right)^{-1}\right\}\right) \\
& \times\left(\left|x_{1}-p+\tau\right|+\left|x_{2}-s+h\right|+\tau+h\right)^{\beta} d s d p d \lambda \\
& +\int_{1 /\left(\tau^{2}+h^{2}\right)}^{\infty} \int_{0}^{\infty} \int_{-\infty}^{\infty}\left(e^{-a(1+\lambda)^{1 / 2}\left(\left|x_{1}-p\right|+\left|x_{2}-s\right|\right)}\right. \\
& \left.\times\left(1+\ln \left\{1+\left((\lambda+1)^{1 / 2}\left(\left|x_{1}-p\right|+\left|x_{2}-s\right|\right)\right)^{-1}\right\}\right)\right) /\left(\left(\tau^{2}+h^{2}\right)^{\alpha+\beta / 2} \lambda^{\alpha}\right) \\
& \left.\times\left(\left|x_{1}-p\right|^{2}+\left|x_{2}-s\right|^{2}\right)^{\beta / 2} d s d p d \lambda\right] \\
\leq & M_{45}(a)\left[\int_{1 /\left(\tau^{2}+h^{2}\right)}^{\infty} \frac{1}{\left(\tau^{2}+h^{2}\right)^{\alpha+\beta / 2} \lambda^{\alpha+\beta / 2+1}} d \lambda+\int_{1 /\left(\tau^{2}+h^{2}\right)}^{\infty} \frac{\tau+h}{\left(\tau^{2}+h^{2}\right)^{\alpha+\beta / 2} \lambda^{\alpha+1}} d \lambda\right] \\
\leq & M_{46}(a) .
\end{aligned}
$$

Thus, for $\tau^{2}+h^{2}<1$, we have

$$
\left|\frac{f\left(x_{1}+\tau, x_{2}+h\right)-f\left(x_{1}, x_{2}\right)}{\left(\tau^{2}+h^{2}\right)^{\alpha+\beta / 2}}\right| \leq M_{47}(a)\|f\|_{E_{\alpha}} .
$$


Next, let us assume that $\tau^{2}+h^{2} \geq 1$. Using formula (36), estimate (3), the triangle inequality, Hilbert's inequality, and the assumption $\tau^{2}+h^{2} \geq 1$, we get

$$
\begin{aligned}
\left|\frac{f\left(x_{1}+\tau, x_{2}+h\right)-f\left(x_{1}, x_{2}\right)}{\left(\tau^{2}+h^{2}\right)^{\alpha+\beta / 2}}\right| & \\
\leq & \int_{0}^{\infty} \int_{0}^{\infty} \int_{-\infty}^{\infty} \frac{\left|G\left(x_{1}+\tau, x_{2}+h, p, s ; \lambda\right)\right|}{\lambda^{\alpha}}\left|\lambda^{\alpha} A(A+\lambda)^{-1} f(p, s)\right| d s d p d \lambda \\
& +\int_{0}^{\infty} \int_{0}^{\infty} \int_{-\infty}^{\infty} \frac{\left|G\left(x_{1}, x_{2}, p, s ; \lambda\right)\right|}{\lambda^{\alpha}}\left|\lambda^{\alpha} A(A+\lambda)^{-1} f(p, s)\right| d s d p d \lambda \\
\leq & M_{48}\|f\|_{E_{\alpha}}\left[\int_{0}^{\infty} \int_{0}^{\infty} \int_{-\infty}^{\infty} \frac{1}{\lambda^{\alpha}} e^{-a(1+\lambda)^{1 / 2}\left(\left|x_{1}+\tau-p\right|+\left|x_{2}+h-s\right|\right)}\right. \\
& \times\left(1+\ln \left\{1+\left((1+\lambda)^{1 / 2}\left(\left|x_{1}+\tau-p\right|+\left|x_{2}+h-s\right|\right)\right)^{-1}\right\}\right) d s d p d \lambda \\
& +\int_{0}^{\infty} \int_{0}^{\infty} \int_{-\infty}^{\infty} \frac{1}{\lambda^{\alpha}} e^{-a(1+\lambda)^{1 / 2}\left(\left|x_{1}-p\right|+\left|x_{2}-s\right|\right)} \\
& \left.\times\left(1+\ln \left\{1+\left((1+\lambda)^{1 / 2}\left(\left|x_{1}-p\right|+\left|x_{2}-s\right|\right)\right)^{-1}\right\}\right) d s d p d \lambda\right] \\
\leq & M_{49}(a)\|f\|_{E_{\alpha}} \int_{0}^{\infty} \frac{1}{\lambda^{\alpha}(1+\lambda)} d \lambda \leq \frac{M_{50}(a)}{\alpha(1-\alpha)}\|f\|_{E_{\alpha} .} .
\end{aligned}
$$

Hence, for $\tau^{2}+h^{2} \geq 1$, we obtain

$$
\left|\frac{f\left(x_{1}+\tau, x_{2}+h\right)-f(t, x)}{\left(\tau^{2}+h^{2}\right)^{\alpha+\beta / 2}}\right| \leq \frac{M_{50}(a)}{\alpha(1-\alpha)}\|f\|_{E_{\alpha}} .
$$

Combining estimates (37) and (38), we get

$$
\sup _{\lambda>0} \sup _{\substack{\left(x_{1}+\tau, x_{2}+h\right),\left(x_{1}, x_{2}\right) \in \mathbb{R}_{+}^{2} \\(\tau, h) \neq(0,0)}}\left|\frac{f\left(x_{1}+\tau, x_{2}+h\right)-f\left(x_{1}, x_{2}\right)}{\left(\tau^{2}+h^{2}\right)^{\alpha+\beta / 2}}\right| \leq \frac{M_{51}(a)}{\alpha(1-\alpha)}\|f\|_{E_{\alpha}} .
$$

Estimates (35) and (39) yield that

$$
E_{\alpha}=E_{\alpha}\left(A, C^{\beta}\left(\mathbb{R}_{+}^{2}\right)\right) \subset C^{2 \alpha+\beta}\left(\mathbb{R}_{+}^{2}\right) .
$$

This is the end of the proof of Theorem 5 .

\section{Applications}

In this section, we consider some applications of Theorem 5. First, we consider the boundary value problem for the elliptic equation

$$
\left\{\begin{array}{l}
-\frac{\partial^{2} u\left(y, x_{1}, x_{2}\right)}{\partial y^{2}}-a_{11}\left(x_{1}, x_{2}\right) \frac{\partial^{2} u\left(y, x_{1}, x_{2}\right)}{\partial x_{1}^{2}}-a_{22}\left(x_{1}, x_{2}\right) \frac{\partial^{2} u\left(y, x_{1}, x_{2}\right)}{\partial x_{2}^{2}}+\sigma u\left(y, x_{1}, x_{2}\right) \\
\quad=f\left(y, x_{1}, x_{2}\right), \quad 0<y<T, x_{1} \in \mathbb{R}^{+}, x_{2} \in \mathbb{R}, \\
u\left(0, x_{1}, x_{2}\right)=\varphi\left(x_{1}, x_{2}\right), \quad u\left(T, x_{1}, x_{2}\right)=\psi\left(x_{1}, x_{2}\right), \quad x_{1} \in \mathbb{R}^{+}, x_{2} \in \mathbb{R}, \\
u\left(y, 0, x_{2}\right)=0, \quad 0 \leq y \leq T, x_{2} \in \mathbb{R} .
\end{array}\right.
$$


Here, $a_{11}\left(x_{1}, x_{2}\right), a_{22}\left(x_{1}, x_{2}\right), \varphi\left(x_{1}, x_{2}\right), \psi\left(x_{1}, x_{2}\right)$, and $f\left(y, x_{1}, x_{2}\right)$ are given smooth functions and they satisfy every compatibility condition and

$$
a_{11}^{2}\left(x_{1}, x_{2}\right)+a_{22}^{2}\left(x_{1}, x_{2}\right) \geq \delta>0
$$

and $\sigma>0$, which guarantees that problem (40) has a smooth solution $u\left(y, x_{1}, x_{2}\right)$.

Theorem 6 For the solution of boundary value problem (40), we have the following estimate:

$$
\begin{aligned}
\left\|u_{y y}\right\|_{C\left(C^{2 \alpha+\beta}\left(\mathbb{R}_{+}^{2}\right)\right)}+\left\|u_{x_{1} x_{1}}\right\|_{C\left(C^{2 \alpha+\beta}\left(\mathbb{R}_{+}^{2}\right)\right)}+\left\|u_{x_{2} x_{2}}\right\|_{C\left(C^{2 \alpha+\beta}\left(\mathbb{R}_{+}^{2}\right)\right)} \\
\leq M(\alpha, \beta)\left[\left\|\varphi_{x_{1} x_{1}}\right\|_{C^{2 \alpha+\beta}\left(\mathbb{R}_{+}^{2}\right)}+\left\|\varphi_{x_{2} x_{2}}\right\|_{C^{2 \alpha+\beta}\left(\mathbb{R}_{+}^{2}\right)}+\left\|\psi_{x_{1} x_{1}}\right\|_{C^{2 \alpha+\beta}\left(\mathbb{R}_{+}^{2}\right)}\right. \\
\left.\quad+\left\|\psi_{x_{2} x_{2}}\right\|_{C^{2 \alpha+\beta}\left(\mathbb{R}_{+}^{2}\right)}+\|f\|_{C\left(C^{2 \alpha+\beta}\left(\mathbb{R}_{+}^{2}\right)\right)}\right],
\end{aligned}
$$

where $M(\alpha, \beta)$ is independent of $\varphi, \psi$, and $f$.

Proof We introduce the Banach space $\mathbb{C}([0, T], E)$ of all continuous abstract functions $u(y)$ defined on $[0, T]$ with values in $E$, equipped with the norm

$$
\|u\|_{\mathbb{C}([0, T], E)}=\max _{0 \leq y \leq T}\|u(y)\|_{E}
$$

Note that problem (40) can be written in the form of the abstract boundary value problem

$$
-\frac{d^{2} u(y)}{d y^{2}}+A u(y)=f(y), \quad 0<y<T, \quad u(0)=\varphi, \quad u(T)=\psi
$$

in a Banach space $E=C\left(\mathbb{R}_{+}^{2}\right)$ with a positive operator $A$ defined by (1). Here $f(y)=$ $f\left(y, x_{1}, x_{2}\right)$ is the given abstract function defined on $[0, T]$ with values in $E, \varphi=\varphi\left(x_{1}, x_{2}\right)$, $\psi=\psi\left(x_{1}, x_{2}\right)$ are elements of $D(A)$. Therefore, the proof of Theorem 6 is based on Theorem 5 on the structure of the fractional spaces $E_{\alpha}\left(A, C^{\beta}\left(\mathbb{R}_{+}^{2}\right)\right)$, Theorem 4 on the positivity of the operator $A$, on the following theorems on coercive stability of elliptic problems, nonlocal boundary value for the abstract elliptic equation and on the structure of the fractional space $E_{\alpha}^{\prime}=E_{\alpha}\left(A^{1 / 2}, E\right)$. This is the end of the proof of Theorem 6 .

Theorem 7 Under assumption (41) for the solution of elliptic problem

$$
\left\{\begin{array}{l}
-a_{11}\left(x_{1}, x_{2}\right) \frac{\partial^{2} u\left(x_{1}, x_{2}\right)}{\partial x_{1}^{2}}-a_{22}\left(x_{1}, x_{2}\right) \frac{\partial^{2} u\left(x_{1}, x_{2}\right)}{\partial x_{2}^{2}}+\sigma u\left(x_{1}, x_{2}\right)=g\left(x_{1}, x_{2}\right), \\
\quad x_{1} \in \mathbb{R}^{+}, x_{2} \in \mathbb{R} \\
u\left(0, x_{2}\right)=0, \quad x_{2} \in \mathbb{R},
\end{array}\right.
$$

the following coercive inequality holds:

$$
\left\|\frac{\partial^{2} u}{\partial x_{1}^{2}}\right\|_{C^{\mu}\left(\mathbb{R}_{+}^{2}\right)}+\left\|\frac{\partial^{2} u}{\partial x_{2}^{2}}\right\|_{C^{\mu}\left(\mathbb{R}_{+}^{2}\right)} \leq M(\mu)\|g\|_{C^{\mu}\left(\mathbb{R}_{+}^{2}\right)}
$$

where $M(\mu)(0<\mu<1)$ does not depend on $g$. 
The proof of Theorem 7 uses the techniques introduced in [5, Chapter 5] and it is based on estimates (3) and (4).

Theorem 8 ([5, Theorem 5.2.48]) The spaces $E_{\alpha}(A, E)$ and $E_{2 \alpha}^{\prime}\left(A^{1 / 2}, E\right)$ coincide for any $0<\alpha<\frac{1}{2}$, and their norms are equivalent.

Theorem 9 ([28, Theorem 3.1]) Let $A$ be a positive operator in a Banach space $E$ and $f \in C\left([0, T], E_{\alpha}^{\prime}\right)(0<\alpha<1)$. Then, for the solution of nonlocal boundary value problem (42), the coercive inequality

$$
\begin{aligned}
& \left\|u^{\prime \prime}\right\|_{C\left([0, T], E_{\alpha}^{\prime}\right)}+\|A u\|_{C\left([0, T], E_{\alpha}^{\prime}\right)} \\
& \quad \leq M\left[\|A \varphi\|_{E_{\alpha}^{\prime}}+\|A \psi\|_{E_{\alpha}^{\prime}}+\frac{M}{\alpha(1-\alpha)}\|f\|_{C\left([0, T], E_{\alpha}^{\prime}\right)}\right]
\end{aligned}
$$

holds, where $M$ does not depend on $\alpha, \varphi, \psi$, and $f$.

Second, we consider the nonlocal boundary value problem for the elliptic equation under assumption (41)

$$
\left\{\begin{array}{l}
-\frac{\partial^{2} u\left(y, x_{1}, x_{2}\right)}{\partial y^{2}}-a_{11}\left(x_{1}, x_{2}\right) \frac{\partial^{2} u\left(y, x_{1}, x_{2}\right)}{\partial x_{1}^{2}}-a_{22}\left(x_{1}, x_{2}\right) \frac{\partial^{2} u\left(y, x_{1}, x_{2}\right)}{\partial x_{2}^{2}}+\sigma u\left(y, x_{1}, x_{2}\right) \\
\quad=f\left(y, x_{1}, x_{2}\right), \quad 0<y<T, x_{1} \in \mathbb{R}^{+}, x_{2} \in \mathbb{R}, \\
u\left(0, x_{1}, x_{2}\right)=u\left(T, x_{1}, x_{2}\right), \quad u_{y}\left(0, x_{1}, x_{2}\right)=u_{y}\left(T, x_{1}, x_{2}\right), \quad x_{1} \in \mathbb{R}^{+}, x_{2} \in \mathbb{R}, \\
u\left(y, 0, x_{2}\right)=0, \quad 0 \leq y \leq T, x_{2} \in \mathbb{R} .
\end{array}\right.
$$

Here, $a_{11}\left(x_{1}, x_{2}\right), a_{22}\left(x_{1}, x_{2}\right)$, and $f\left(y, x_{1}, x_{2}\right)$ are given smooth functions and they satisfy every compatibility condition and (41), which guarantees that problem (43) has a smooth solution $u\left(y, x_{1}, x_{2}\right)$.

Theorem 10 For the solution of initial boundary value problem (43), we have the following estimate:

$$
\begin{aligned}
& \left\|u_{y y}\right\|_{C\left(C^{2 \alpha+\beta}\left(\mathbb{R}_{+}^{2}\right)\right)}+\left\|u_{x_{1} x_{1}}\right\|_{C\left(C^{2 \alpha+\beta}\left(\mathbb{R}_{+}^{2}\right)\right)}+\left\|u_{x_{2} x_{2}}\right\|_{C\left(C^{2 \alpha+\beta}\left(\mathbb{R}_{+}^{2}\right)\right)} \\
& \quad \leq M(\alpha, \beta)\|f\|_{C\left(C^{2 \alpha+\beta}\left(\mathbb{R}_{+}^{2}\right)\right)}
\end{aligned}
$$

where $M(\alpha, \beta)$ is independent off .

The proof of Theorem 10 is based on Theorem 5 on the structure of the fractional spaces $E_{\alpha}\left(A, C^{\beta}\left(\mathbb{R}_{+}^{2}\right)\right)$, Theorem 4 on the positivity of the operator $A$, Theorem 7 on coercive stability of the elliptic problem, Theorem 8 on the structure of the fractional space $E_{\alpha}^{\prime}=$ $E_{\alpha}\left(A^{1 / 2}, E\right)$, and the following theorem on coercive stability of the nonlocal boundary value for the abstract elliptic equation.

Theorem 11 ([28, Theorem 3.1]) Let $A$ be a positive operator in a Banach space $E$ and $f \in C\left([0, T], E_{\alpha}^{\prime}\right)(0<\alpha<1)$. Then, for the solution of nonlocal boundary value problem (40)

$$
\left\{\begin{array}{l}
-u^{\prime \prime}(y)+A u(y)=f(y), \quad 0<y<T, \\
u(0)=u(T), \quad u^{\prime}(0)=u^{\prime}(T)
\end{array}\right.
$$


in a Banach space $E$ with a positive operator $A$, the coercive inequality

$$
\left\|u^{\prime \prime}\right\|_{C\left([0, T], E_{\alpha}^{\prime}\right)}+\|A u\|_{C\left([0, T], E_{\alpha}^{\prime}\right)} \leq \frac{M}{\alpha(1-\alpha)}\|f\|_{C\left([0, T], E_{\alpha}^{\prime}\right)}
$$

holds, where $M$ does not depend on $\alpha$ and $f$.

\section{Conclusion}

In the present article, the structure of the fractional spaces $E_{\alpha}\left(A, C^{\beta}\left(\mathbb{R}_{+}^{2}\right)\right)$ generated by the two-dimensional elliptic differential operator $A$ is investigated. The positivity of this operator $A$ in a Hölder space is established. Of course, the Banach fixed point theorem and the method of the present paper enable us to establish the existence and uniqueness results which hold under some sufficient conditions on the nonlinear term for the solution of the mixed problem

$$
\left\{\begin{array}{l}
-\frac{\partial^{2} u\left(y, x_{1}, x_{2}\right)}{\partial y^{2}}-a_{11}\left(x_{1}, x_{2}\right) \frac{\partial^{2} u\left(y, x_{1}, x_{2}\right)}{\partial x_{1}^{2}}-a_{22}\left(x_{1}, x_{2}\right) \frac{\partial^{2} u\left(y, x_{1}, x_{2}\right)}{\partial x_{2}^{2}}+\sigma u\left(y, x_{1}, x_{2}\right) \\
\quad=f\left(y, x_{1}, x_{2}, u, u_{x_{1}}, u_{x_{2}}, u_{y}\right), \quad 0<y<T, x_{1} \in \mathbb{R}^{+}, x_{2} \in \mathbb{R}, \\
u\left(0, x_{1}, x_{2}\right)=\varphi\left(x_{1}, x_{2}\right), \quad u\left(T, x_{1}, x_{2}\right)=\psi\left(x_{1}, x_{2}\right), \quad x_{1} \in \mathbb{R}^{+}, x_{2} \in \mathbb{R}, \\
u\left(y, 0, x_{2}\right)=0, \quad 0<y<T, x_{2} \in \mathbb{R} .
\end{array}\right.
$$

\section{Competing interests}

The authors declare that they have no competing interests.

\section{Authors' contributions}

All authors read and approved the final manuscript.

\section{Author details}

${ }^{1}$ Department of Mathematics, Fatih University, 34500 Buyukcekmece, Istanbul, Turkey. ${ }^{2}$ Department of Mathematics, ITTU, Ashgabat, Turkmenistan. ${ }^{3}$ Present address: Hacettepe Universitesi, Fen Fakultesi, Matematik Bolumu, 06800 Beytepe, Ankara, Turkey.

\section{Acknowledgements}

Some of the results of the present article were announced in the conference proceeding [11] as an extended abstract without proofs. The authors would like to thank Prof. Pavel Sobolevskii (Universidade Federal do Ceará, Brasil). The second author would also like to thank The Scientific and Technological Research Council of Turkey (TUBITAK) for the financial support.

Received: 30 October 2013 Accepted: 3 December 2013 Published: 02 Jan 2014

\section{References}

1. Fattorini, HO: Second Order Linear Differential Equations in Banach Spaces. Mathematics Studies. North-Holland, Amsterdam (1985)

2. Grisvard, P: Elliptic Problems in Nonsmooth Domains. Pitman, London (1984)

3. Krein, SG: Linear Differential Equations in a Banach Space. Translations of Mathematical Monographs. Am. Math. Soc., Providence (1968)

4. Ashyralyev, A, Sobolevskii, PE: Well-Posedness of Parabolic Difference Equations. Birkhäuser, Basel (1994)

5. Ashyralyev, A, Sobolevskii, PE: New Difference Schemes for Partial Differential Equations. Birkhäuser, Basel (2004)

6. Krasnosel'skii, MA, Zabreiko, PP, Pustyl'nik, El, Sobolevskii, PE: Integral Operators in Spaces of Summable Functions. Noordhoff, Leiden (1976)

7. Agarwal, R, Bohner, M, Shakhmurov, VB: Maximal regular boundary value problems in Banach-valued weighted spaces. Bound. Value Probl. 1, 9-42 (2005)

8. Alibekov, KhA, Sobolevskii, PE: Stability of difference schemes for parabolic equations. Dokl. Akad. Nauk SSSR 232(4), 737-740 (1977)

9. Alibekov, KhA: Investigations in $C$ and $L_{p}$ of difference schemes of high order accuracy for approximate solutions of multidimensional parabolic boundary value problems. Dissertation. Voronezh State University, Voronezh (1978)

10. Alibekov, KhA, Sobolevskii, PE: Stability and convergence of difference schemes of a high order for parabolic differential equations. Ukr. Mat. Zh. 31(6), 627-634 (1979)

11. Ashyralyev, A, Akturk, S, Sozen, Y: Positivity of two-dimensional elliptic differential operators in Hölder space. AIP Conf. Proc. 1470, 77-79 (2012) 
12. Danelich, SI: Fractional powers of positive difference operators. Dissertation. Voronezh State University, Voronezh (1989)

13. Lunardi, A: Analytic Semigroups and Optimal Regularity in Parabolic Problems. Birkhäuser, Basel (1995)

14. Simirnitskii, YuA, Sobolevskii, PE: Positivity of multidimensional difference operators in the C-norm. Usp. Mat. Nauk 36(4), 202-203 (1981)

15. Sobolevskii, PE: The coercive solvability of difference equations. Dokl. Akad. Nauk SSSR, 201(5), 1063-1066 (1980)

16. Solomyak, MZ: Analytic semigroups generated by elliptic operator in spaces $L_{p}$. Dokl. Akad. Nauk SSSR, 127(1), 37-39 (1959)

17. Solomyak, MZ: Estimation of norm of the resolvent of elliptic operator in spaces $L_{p}$. Usp. Mat. Nauk 15(6), 141-148 (1960)

18. Stewart, HB: Generation of analytic semigroups by strongly elliptic operators under general boundary conditions. Trans. Am. Math. Soc. 259, 299-310 (1980)

19. Ashyralyev, A: Fractional spaces generated by the positive differential and difference operators in a Banach space. In: Tas, K, Tenreiro Machado, JA, Baleanu, D (eds.) Mathematical Methods in Engineering. Springer, Dordrecht (2007)

20. Hardy, GH, Littlewood, JE, Pólya, G: Inequalities. Cambridge University Press, Cambridge (1988)

21. Ashyralyev, A, Sobolevskii, PE: The linear operator interpolation theory and the stability of the difference schemes. Dokl. Akad. Nauk SSSR, 275(6), 1289-1291 (1984)

22. Triebel, H: Interpolation Theory, Function Spaces, Differential Operators. North-Holland, Amsterdam (1978)

23. Ashyralyev, A: Method of positive operators of investigations of the high order of accuracy difference schemes for parabolic and elliptic equations. Dissertation, Inst. of Math. of Acad. Sci. Kiev (1992)

24. Ashyralyev, A, Yaz, N: On structure of fractional spaces generated by positive operators with the nonlocal boundary value conditions. In: Agarwal, RP (ed.) Proceedings of the Conference Differential and Difference Equations and Applications. Hindawi Publishing Corporation, New York (2006)

25. Ashyralyev, A, Tetikoğlu, FS: The structure of fractional spaces generated by the positive operator with periodic conditions. In: AIP Conference Proceedings. ICAAM 2012, vol. 1470, pp. 57-60 (2012)

26. Bazarov, MA: On the structure of fractional spaces. In: Proceedings of the XXVII All-Union Scientific Student Conference 'The Student and Scientific-Technological Progress', pp. 3-7. Novosibirsk. Gos. Univ., Novosibirsk (1989) (in Russian)

27. Ashyralyev, A, Nalbant, N, Sozen, Y: Structure of fractional spaces generated by second order difference operators. J. Franklin Inst. (2013). doi:10.1016/j.jfranklin.2013.07.009

28. Ashyralyev, A: On well-posedness of the nonlocal boundary value problems for elliptic equations. Numer. Funct. Anal. Optim. 24, 1-15 (2003)

10.1186/1687-2770-2014-3

Cite this article as: Ashyralyev et al.: The structure of fractional spaces generated by a two-dimensional elliptic differential operator and its applications. Boundary Value Problems 2014, 2014:3

\section{Submit your manuscript to a SpringerOpen ${ }^{\odot}$ journal and benefit from:}

- Convenient online submission

Rigorous peer review

Immediate publication on acceptance

- Open access: articles freely available online

- High visibility within the field

- Retaining the copyright to your article 